\title{
Automated Analysis of Directional Optical Coherence Tomography Images
}

\author{
Florence Rossant ${ }^{1(\bowtie)}$, Kate Grieve ${ }^{2}$, and Michel Paques ${ }^{2}$ \\ ${ }^{1}$ Institut Supérieur d'Electronique de Paris (ISEP), Paris, France \\ Florence.rossant@isep.fr \\ ${ }^{2}$ Clinical Investigation Center 1423, Quinze-Vingts Hospital, Paris, France \\ kategrieve@gmail.com, michel.paques@gmail.com
}

\begin{abstract}
Directional optical coherence tomography (D-OCT) reveals reflectance properties of retinal structures by changing the incidence angle of the light beam. As no commercially available OCT device has been designed for such use, image processing is required to homogenize the grey levels between off-axis images before differential analysis. We describe here a method for automated analysis of D-OCT images and propose a color representation to highlight angle-dependent structures. Clinical results show that the proposed approach is robust and helpful for clinical interpretation.
\end{abstract}

Keywords: Optical coherence tomography · Directional OCT (D-OCT) • Retina $\cdot$ Ophthalmology $\cdot$ Differential analysis $\cdot$ Markov random fields

\section{Introduction}

Photoreceptors have unique optical properties, one of which is the angular dependence of their absorbance and reflectance, known as the Stiles-Crawford effect (SCE) [1, 2]. As a result, the distribution of backscattered light through the pupil is modulated by its incidence. The angle-dependent absorbance and reflectance of individual cone photoreceptors follows a Gaussian curve, with a peak whose orientation defines the photoreceptor pointing and whose acceptance angle correlates with the span of photon capture. Cones account for most of the SCE.

Gao et al. first used a custom OCT setup to image off-axis macular photoreceptors and measure the contributions of macular photoreceptor substructures to the optical SCE (oSCE) [3]. They suggested that directional reflectance of the cone outer segment tip (COST) line and, to a lesser extent, of the inner/outer segment junction (IS/OS), accounts for most of the oSCE of macular photoreceptors. This was supported by the findings of Miloudi et al. who documented the directional reflectance of photoreceptors using a combined approach by en face adaptive optics imaging and D-OCT [4]. The potential medical interest of D-OCT was suggested by Lujan et al. who used D-OCT to delineate the Henle fiber layer, that is, the photoreceptor axons, and subsequently extract the thickness of the outer nuclear layer [5]. In addition, our group and others have recently demonstrated differences due to pathology in the photoreceptor layers using D-OCT in patients (Pedinielli et al. IOVS 2016: ARVO E-Abstract 4248, Lujan et al. IOVS 2016: ARVO E-Abstract 4250). Hence, D-OCT appears to be an interesting 
approach to document subtle retinal changes. We describe here a method for automated analysis of D-OCT images acquired with commercial OCT systems. We propose an efficient visualization of D-OCT data that helps develop the clinical interpretation by highlighting angle-dependent structures.

\section{Methods}

D-OCT scans were acquired using a commercially available OCT apparatus (Spectralis ${ }^{\circledR}$ SD-OCT). A reference scan was acquired in the standard mode, with a light beam parallel to the visual axis. Two off-axis images were also acquired, based on a manual procedure, at approximately equal $2^{\circ}$ to $3^{\circ}$ angles $\alpha$ to the right and left of the visual axis. All images were aligned by the acquisition system (Fig. 1).



(a) $I_{1}$



(b) $I_{2}$

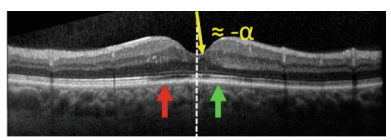

(c) $I_{3}$

Fig. 1. A set of two off-axis images (a) (c) along with a standard on-axis acquisition (b), all aligned. Red and green arrows show evidence of anisotropy. (Color figure online)

Anisotropy properties of the reflectance can be brought out by computing the difference between the two off-axis images. Retinal structures that have similar reflectance responses whatever the light incidence angle are considered isotropic and lead to values near zero. On the contrary, anisotropic areas lead to positive or negative values, depending on the local orientation of the retinal structures with respect to the incidence angle. However the simple difference of the two angled images is not sufficient here. Indeed, the global brightness of the image is no longer homogeneous over the entire image when the operator turns the light beam away from the visual axis (Fig. 1(a) and (c)). Note that the left side is darker than the right side for the angle $\alpha$ and conversely for the opposite angle $-\alpha$. So the basic difference of the two off-axis images cannot provide suitable enhancement of anisotropic features (Fig. 2).



Fig. 2. The absolute difference of the two input off-axis images.

Consequently, we have developed dedicated image processing methods to compensate for illumination inhomogeneity, before performing differential analysis. The proposed method relies on the segmentation of the retinal area (Sect. 3), followed by an illumination correction applied on this area (Sect. 4). We do not have prior knowledge 
of the physical model underlying this non-uniform illumination of the off-axis images, as we use the OCT acquisition system in an unconventional manner. However, we observe that the overall illumination basically varies along the horizontal axis. Therefore, our correction models rely on functions of the horizontal x-coordinate. We also propose post-processing algorithms to achieve a colored representation of anisotropic areas (Sect. 5). To the best of our knowledge only one article [6] presents image processing methods for D-OCT analysis. However, a global correction of the overall brightness is applied on each image separately, which does not allow one to deal with cases like the one presented in Fig. 2.

Let us denote by $I_{1}(x, y)$ and $I_{3}(x, y)$ the two off-axis images and by $I_{2}(x, y)$ the standard image. The grayscale values are coded by floating point numbers in $[0,1]$.

\section{Retinal Segmentation and Estimation of the Incidence Angle}

Segmentation is necessary to determine the area over which the illumination correction has to be applied. We apply the method of [7,8] to determine the region comprised between the inner limiting membrane (ILM) and the external interface of the hyper reflective complex (HRC), which is also the outer edge of the retinal pigment epithelium (RPE). Horizontally, we restrict the region of interest (ROI) to the interval $\left[x_{L}, x_{R}\right], 6 \mathrm{~mm}$ wide, centered on the foveola $x_{F}$ (Fig. 3).


Fig. 3. Segmentation of the retinal data and definition of the ROI.

The beam incidence is manually and approximately set by the operator, as no commercially available OCT apparatus has been designed for such use. Hence, it is interesting to calculate the beam angle $\alpha$ from the image itself. We dramatically increase the contrast in the vitreous and the choroid (Fig. 4(a)) and apply a morphological closing to simplify the image. These operations reveal the main edges, in particular the contours of the rectangular image that is tilted off by the sought for angle $\alpha$ (see the upper right black triangle). We extract the edges (Canny operator) and apply a Hough transform so as to obtain the main linear segments (Fig. 4(b)). We compute the angle $\theta(j)$ and the length $l(j)$ of each extracted segment $j=1, \ldots J$. For each angle $\theta(j)$, we calculate the sum of the lengths of all segments having the same orientation up to $2^{\circ}(1)$. The angle with the highest cumulative length $L$ is the incident angle $\alpha$.

$$
L(j)=\sum_{i \in E(j)} l(i), E(j)=\left\{i \in\{1, \ldots, J\} /|\theta(i)-\theta(j)|<2^{\circ}\right\}
$$



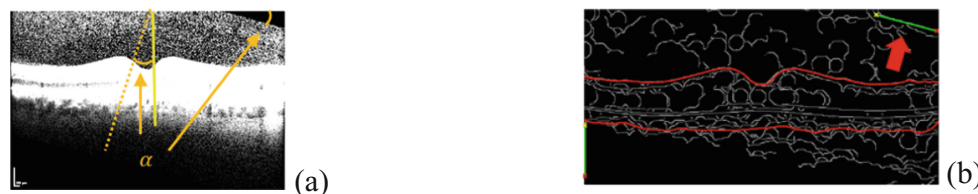

Fig. 4. Estimation of the incident light beam angle from the saturated image (a); linear segments (in green) extracted from the contour image through the Hough transform (b). (Color figure online)

\section{Illumination Correction}

\subsection{Individual Illumination Correction}

A first the illumination correction is independently applied to both off-axis images to better balance their overall intensity. We divide the input image $I(x, y)$ (actually $I_{1}(x, y)$ or $I_{3}(x, y)$, Fig. 5(a)) into two parts, by slicing it vertically at the foveola $x_{F}$. We classify the pixels of the retinal area into 3 classes with a k-means algorithm $(k=3$ classes), applied to both sub-images independently (Fig. 5(b)). Let us denote by $c_{k}^{(L)}$, $c_{k}^{(R)}, k=1,2,3$, the centers of the clusters for the left $(\mathrm{L})$ and right $(\mathrm{R})$ sub-images. These values represent the mean intensity of each cluster. We use them to define straight lines that represent a linear estimation of the intensity variation of the actual value $I_{k}^{(0)}$ of cluster $k$ with respect to the $x$ coordinate (Fig. 5(b)).



(a) $I_{1}$



(c) $I_{1}^{\prime}$


(b)

Fig. 5. Individual correction of the intensity of off-axis images; (b) K-means classification applied to input image (a), and linear estimation of the intensity variation; (c) Corrected image.

The two points defining each of the three lines are set at the middle $x$ coordinate of every half image, and vertically take the intensity value of the cluster. We propose modeling the variation of the intensity $I_{k}^{(0)}$ along the horizontal axis as follows: 
F. Rossant et al.

$$
I_{k}(x, y)=I_{k}^{(0)}+a \frac{x-x_{F}}{x_{r}-x_{l}} I_{k}^{(0)}+b \frac{x-x_{F}}{x_{r}-x_{l}}
$$

The parameters $a$ and $b$ are estimated from points $\left(x_{l}, c_{k}^{(L)}\right)$ and $\left(x_{r}, c_{k}^{(R)}\right)$, $k=1,2,3$, by minimizing the mean quadratic error at these six points. We deduce from (2) the transform to apply to each pixel $(x, y)$ of intensity $I(x, y)$ :

$$
I^{\prime}(x, y)=\left[I(x, y)-b \frac{x-x_{F}}{x_{r}-x_{l}}\right] /\left[1+a \frac{x-x_{F}}{x_{r}-x_{l}}\right]
$$

So, the overall intensity is better balanced over the corrected off-axis images, $I_{1}^{\prime}$ and $I_{3}^{\prime}$, which are then normalized in $[0,1]$ by linear contrast stretching (Fig. 5(c)).

\subsection{Joint Illumination Correction}

The corrected images are then jointly processed in the ROI. We propose modeling the illumination correction as 3rd order polynomial function of the $\mathrm{x}$-coordinate:

$$
f(x)=a x^{3}+b x^{2}+c x+d
$$

The purpose of the optimization is to define the parameters $a, b, c, d$ of this function. The corrected image is defined by

$$
I_{3}^{\prime \prime}(x, y)=f(x) I_{3}^{\prime}(x, y)
$$

The optimization process consists of minimizing the number of pixels of the ROI that significantly differ between the first off-axis image $I_{1}^{\prime}$, viewed as the reference image, and the corrected off-axis image $I_{3}^{\prime \prime}$. So we define the following criterion:

$$
C(a, b, c, d)=\operatorname{card}\left\{(x, y) \in R O I /\left|I_{1}^{\prime}(x, y)-I_{3}^{\prime \prime}(x, y)\right| \geq T\right\}
$$

where $T$ is a threshold dynamically set from the source images, equal to twice the standard deviation of the difference image $\left|I_{1}^{\prime}-I_{3}^{\prime}\right|$. The optimal parameters of the correction function $f$ are obtained by minimizing the criterion $C$ :

$$
\left(a_{\text {opt }}, b_{\text {opt }}, c_{\text {opt }}, d_{\text {opt }}\right)=\operatorname{Arg} \min _{a, b, c, d}\{C(a, b, c, d)\}
$$

The image $I_{3}^{\prime}$ is corrected by Eq. (5) with the optimal parameters of the polynomial function obtained through (7) (Fig. 6). It is worth noticing that the RPE layer gives a zero response in the difference image, which proves retrospectively that our correction model is valid, as this retinal layer is known to be isotropic. 




(a) $I_{1}^{\prime}$



(b) $I_{3}^{\prime \prime}$

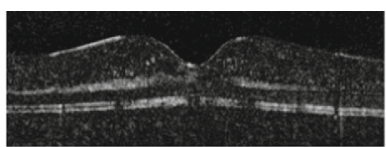

(c) $\left|I_{1}^{\prime}-I_{3}^{\prime \prime}\right|$

Fig. 6. The absolute difference (c) of the two pre-processed off-axis images (a), (b).

\section{Differential Analysis}

\subsection{Color Representation}

A color representation of the anisotropic retinal structures is proposed to indicate the angle of maximal response. We calculate the difference image $I_{D}$ on the ROI:

$$
I_{D}(x, y)=\frac{I_{D 0}(x, y)}{\max _{(x, y)}\left\{\left|I_{D 0}(x, y)\right|\right\}}, \text { where } I_{D 0}(x, y)=\left\{\begin{array}{l}
I_{1}^{\prime}(x, y)-I_{3}^{\prime \prime}(x, y) \text { if }(x, y) \in R O I \\
0 \text { otherwise }
\end{array}\right.
$$

The operator sets a threshold $S \in[0,1]$ that classifies the pixels $(x, y)$ in two sets: isotropic if $\left|I_{D}(x, y)\right|<S$ and anisotropic otherwise. Two colored images, coded in the RGB color space, are finally derived from this classification. Let us denote by $c \in$ $\{1,2,3\}$ the third coordinate coding the color channel (1 for red, 2 for green and 3 for blue). The first output image $I_{R G}$ shows the anisotropic regions in red and green, with the color coding the angle of maximal response (9). The second output image $I_{R G B}$ provides the same information superimposed on a fusion image, which is defined as the minimum of $I_{1}^{\prime}$ and $I_{3}^{\prime \prime}$. Figure 7 shows the output color images obtained with our example, for $S=0.1$, which is generally a good choice.

$$
\begin{aligned}
& I_{R G}(x, y, 1)=I_{D}(x, y) \text { if } I_{D}(x, y)>S, \quad 0 \text { otherwise } \\
& I_{R G}(x, y, 2)=-I_{D}(x, y) \text { if } I_{D}(x, y)<-S, 0 \text { otherwise } \\
& I_{R G}(x, y, 3)=0
\end{aligned}
$$
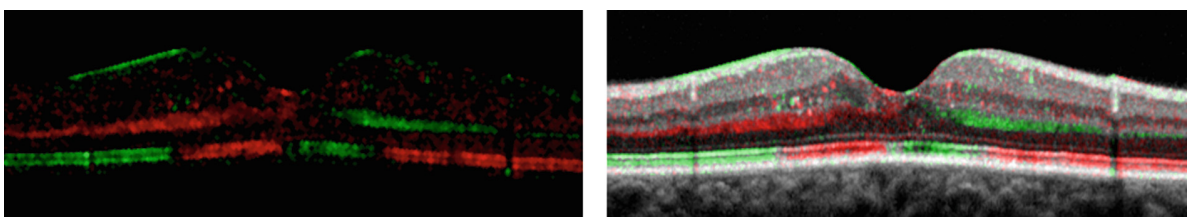

Fig. 7. Color representation of anisotropy: $I_{R G}$ (left), $I_{R G B}$ (right). (Color figure online)

\subsection{Regularization}

The results presented in Fig. 7 can be regularized based on Markov random field (MRF) techniques [9], since the Markovian hypothesis allows us to take into account 
spatial interactions between connected pixels. We define $K=3$ classes corresponding to the regions in red, black and green respectively in $I_{R G}(9)$. The center $\mu_{k}$ of every cluster $k \in\{1,2,3\}$ is initialized to the mean intensity in $I_{D}$ of the pixels belonging to class $k$. We also assume that the noise in the difference image $I_{D}$ follows a Gaussian distribution, and we experimentally set its standard deviation $\sigma_{k}$ to a fixed value, 0.1 . So we have defined the probability distributions $P\left(I_{D_{S}} \mid \omega_{s}=k\right)$ of the pixel intensities (denoted by $I_{D_{S}}$ where $s$ is referred to as a site, i.e. a pixel), conditionally to every class $k$. We refine the classification according to the Bayesian maximum a posteriori (MAP) criterion, i.e. by looking for the label configuration that maximizes the probability of the class field (the labels) conditionally to the observation field (the intensity image $I_{D}$ ). This optimal configuration corresponds to a minimum state of an energy function $U$, defined as follows.

$$
U\left(\omega \mid I_{D}\right)=\sum_{s}\left(\frac{\left(I_{D s}-\mu_{\omega_{s}}\right)^{2}}{2 \sigma_{\omega_{s}}^{2}}+\ln \left(\sqrt{2 \pi} \sigma_{\omega_{s}}\right)\right)+\beta \sum_{(s, t)} \varphi\left(\omega_{s}, \omega_{t}\right)
$$

In this equation, $\varphi\left(\omega_{s}, \omega_{t}\right)$ refers to the Potts model, expressing interactions between the 8-connected sites $s$ and $t$. The first term of (10) is related to the image data, while the second term is a regularization term. The parameter $\beta$, empirically set $(\beta=0.9)$, weights the relative influence of each. The energy function is minimized by running the simulated annealing (SA) algorithm. Figure 8 shows the resulting images.


Fig. 8. Final images $\left(I_{R G}\right.$ and $\left.I_{R G B}\right)$ obtained after RMF based regularization, less noisy than in Fig. 7, showing abnormal distribution of anisotropy of outer retinal structures.

\section{Experimental Results and Conclusion}

We have applied the proposed method on a database of 60 sets of D-OCT images, from 33 patients, including normal cases $(31 \%)$ and pathological cases: $27 \%$ of resolved macular edema, $42 \%$ with macular telangiectasia. The study followed international ethical requirements and informed consent was obtained from all patients.

The results presented below (Fig. 9) were obtained automatically with the proposed method, with $S=0.1$. Overall, we observed a good robustness of our algorithm, with about $15 \%$ failure, revealed by noisy results or an anisotropy detected in the RPE. The probable causes of failure are saturation of an off-axis image to black or white, off-axis images that are too noisy, or a significant shift in the position of the vertical or horizontal slice, due to eye movement during acquisition, and causing artefacts. 




(a) Normal



(c) Macular edema



(b) Macular telangiectasia



(d) Macular edema

Fig. 9. Some clinical results. (a) Control patient, with uniform color in each hemiretinas; (b), (c), (d) Pathological cases: disruption of the coloration suggests abnormal distribution of anisotropy. (Color figure online)

Highlighting the anisotropy of outer retinal layers on OCT scans may help to better interpret some abnormalities seen in the photoreceptor layers. A common finding in retinal diseases is indeed the observation of discontinuities of outer retinal layers, which is usually attributed to loss of photoreceptors. Identifying directional variability of the photoreceptor reflectance helps to identify "hidden" photoreceptor outer structures. We have observed areas of outer retinal layers showing a profile of directional reflectance which is clearly different from normal eyes. In particular, color coding enabled detection of adjacent areas with different directions of peak reflectivity (Figs. 8 and 9). This suggests the presence of photoreceptor disarray, that is, changes in the pointing of photoreceptors. The fact that such disarray was detected in very different clinical situations suggests that it is a common process in retinal diseases. D-OCT may help therefore to disambiguate missing from misaligned cones.

We plan now to refine our acquisition protocol and to increase the number of incidence angles in order to study more accurately reflectance properties.

\section{References}

1. Stiles, W.S., Crawford, B.S.: The luminous efficiency of rays entering the eye pupil at different points. Proc. R. Soc. Lond. 112, 428-450 (1933)

2. Westheimer, G.: Directional sensitivity of the retina: 75 years of Stiles-Crawford effect. Proc. Biol. Sci. 275, 2777-2786 (2008)

3. Gao, W., Cense, B., Zhang, Y., et al.: Measuring retinal contributions to the optical Stiles-Crawford effect with optical coherence tomography. Opt. Express 16, 6486-6501 (2008)

4. Miloudi, C., Rossant, F., Bloch, I., et al.: The negative cone mosaic: a new manifestation of the optical stiles-crawford effect in normal eyes. Invest. Ophthalmol. Vis. Sci. 56, 7043-7050 (2015)

5. Lujan, B.J., Roorda, A., Croskrey, J.A., et al.: Directional optical coherence tomography provides accurate outer nuclear layer and Henle fiber layer measurements. Retina 35, 15111520 (2015)

6. Makhijani, V.S., Roorda, A., Bayabo, J.-K., et al.: Chromatic visualization of reflectivity variance within hybridized directional OCT images. In: Proceedings of SPIE 8571, Optical Coherence Tomography and Coherence Domain Optical Methods in Biomedicine XVII, 857105, 20 March, 2013. doi:10.1117/12.2007141 
F. Rossant et al.

7. Ghorbel, I., Rossant, F., Bloch, I., Tick, S., Pâques, M.: Automated segmentation of macular layers in OCT images and quantitative evaluation of performances. Pattern Recogn. 44(8), 1590-1603 (2011)

8. Rossant, F., Bloch, I., Ghorbel, I., Pâques, M.: Parallel double snakes. Application to the segmentation of retinal layers in 2D-OCT for pathological subjects. Pattern Recogn. 48(12), 3857-3870 (2015)

9. Geman, S., Geman, D.: Stochastic relaxation, Gibbs distribution, and the Bayesian restauration of images. IEEE Trans. PAMI 6(6), 721-741 (1984) 